\title{
FITOSSANIDADE
}

\section{EFEITO DE EXTRATOS AQUOSOS DE PLANTAS NA OVIPOSIÇÃO DA TRAÇA-DAS-CRUCÍFERAS, EM COUVE ${ }^{(1)}$}

\author{
CESAR AUGUSTO MANFRÉ MEDEIROS ${ }^{(2)}$; ARLINDO LEAL BOIÇA JUNIOR ${ }^{(3)}$; \\ ADALCI LEITE TORRES ${ }^{(2)}$
}

\begin{abstract}
RESUMO
Avaliou-se o efeito de extratos aquosos de Achillea millefolium L. (folhas), Azadirachta indica A. Juss. (folhas), Bidens pilosa L. (folhas, frutos e ramos), Bougainvillea glabra Choisy (folhas), Chenopodium ambrosioides L. (folhas, frutos e ramos), Datura suaveolens Humb \& Bonpl. ex. Willd (folhas), Enterolobium contortisilliquum (Vell.) Morong (frutos), Mentha crispa L. (folhas e ramos), Nicotiana tabacum L. (folhas), Piper nigrum L. (folhas), Plumbago capensis Thunb. (folhas e ramos), Pothomorphe umbellata L. (folhas), Sapindus saponaria L. (folhas), S. saponaria (frutos), Solanum cernuum Vell. (folhas), Stryphnodendron adstringens (Mart) Coville (casca), Symphytum officinale L. (folhas), Trichilia catigua A. Juss. (folhas), T. catigua (ramos), Trichilia pallida Sw. (folhas) e T. pallida (ramos), em relação à preferência para oviposição de Plutella xylostella. Discos de folhas de couve (Brassica oleracea var. acephala) cultivar Georgia foram imersos em cada extrato à concentração de 10\% (massa/volume) por um minuto. Em seguida, foram divididos em quatro partes iguais e duas partes foram colocadas alternadamente com outras duas partes tratadas com água destilada, em uma gaiola. A contagem dos ovos foi feita após 24 horas. Os extratos apresentaram efeito deterrente na oviposição da praga, com exceção do extrato de S. adstringens, que não diferiu da testemunha, tratada apenas com água destilada. Os extratos de E. contortisilliquum, S. saponaria (frutos) e T. pallida (folhas) foram os mais eficientes, apresentando 100\% de deterrência.
\end{abstract}

Palavras-chave: Insecta, Plutella xylostella, planta inseticida, couve.

\section{ABSTRACT \\ EFFECT OF PLANTS AQUEOUS EXTRACTS ON OVIPOSITION OF THE DIAMONDBACK, IN KALE}

The effect of aqueous extracts from Achillea millefolium L. (leaves), Azadirachta indica A. Juss. (leaves), Bidens pilosa L. (leaves, fruits e branches), Bougainvillea glabra Choisy (leaves), Chenopodium ambrosioides L. (leaves, fruits e branches), Datura suaveolens Humb \& Bonpl. ex. Willd (leaves), Enterolobium contortisilliquum (Vell.) Morong (fruits), Mentha crispa L. (leaves e branches), Nicotiana tabacum L. (leaves), Piper nigrum L. (leaves), Plumbago capensis Thunb. (leaves e branches), Pothomorphe umbellata L. (leaves), Sapindus saponaria

(1) Recebido para publicação em 2 de julho de 2004 e aceito em 10 de janeiro de 2005.

${ }^{2}$ ) Acadêmico do Curso de Pós-Graduação em Agronomia (Entomologia Agrícola) da FCAV/UNESP, Jaboticabal (SP).

$\left({ }^{3}\right)$ Departamento de Fitossanidade, FCAV/UNESP, Via de Acesso Prof. Paulo Donato Castellane, s/n. ${ }^{\circ}$, 14884-900 Jaboticabal (SP). E-mail: aboicajr@fcav.unesp.br. 
L. (leaves), S. saponaria (fruits), Solanum cernuum Vell. (leaves), Stryphnodendron adstringens (Mart) Coville (bark), Symphytum officinale L. (leaves), Trichilia catigua A. Juss. (leaves), T. catigua (branches), Trichilia pallida Sw. (leaves) e T. pallida (branches), was evaluated in relation to oviposition preference of Plutella xylostella. Disks of kale leaves (Brassica oleracea var. acephala), cultivar Georgia were immersed in each extract at a concentration of $10 \%$ (weight/volume) for one minute, and afterwards, divided in four equal parts, and two parts were placed alternately with other two parts treated with distilled water, in each cage. The counting of the eggs was made after 24 hours. The results showed deterrent effect on oviposition of the pest, except for the extract of $S$. adstringens, which didn't differed from the water treated control. The extracts of E. contortisilliquum, S. saponaria (fruits) and T. pallida (leaves) were the most efficient, presenting $100 \%$ of deterrence.

Key words: Insecta, Plutella xylostella, plant insecticide, Cruciferae.

\section{INTRODUÇÃO}

A couve, Brassica oleracea var. acephala, destacase entre as plantas hortícolas como um dos alimentos importantes na nutrição humana, sendo rica em minerais e vitaminas (FRANCO, 1960). É uma cultura atacada por diversas pragas, tais como: pulgões, curuquerê da couve, traça-das-crucíferas, lagarta-rosca e lagarta-mede-palmo (GALlo et al., 2002). MARANHÃO et al. (1998) consideram a traça-das-crucíferas, Plutella xylostella (L. 1758) (Lepidoptera: Plutellidae) a principal praga da couve, repolho e outras brássicas. Destaca-se pela alta taxa de alimentação durante o período larval, causando grandes prejuízos à cultura, chegando a provocar até $100 \%$ de perdas na produção (Ooi e Kelderman, 1979; Villas BôAs et al., 1990; CHeN et al., 1996).

A principal forma de controle dessa praga é o controle químico (VILLAS BôAS et al., 1990; FRANÇA et al., 1985); todavia, o uso indiscriminado de inseticidas pode proporcionar o surgimento de populações de traça-das-crucíferas resistentes.

Produtos naturais extraídos de plantas constituem-se em fonte de substâncias bioativas compatíveis com programas de manejo integrado de pragas (MIP), o que pode reduzir os efeitos negativos ocasionados pela aplicação descontrolada de inseticidas organossintéticos.

Plantas tratadas com produtos derivados de Azadirachta indica, segundo Schmutterer (1990), inibem a oviposição de diversos lepidópteros, dentre os quais, Spodoptera frugiperda (J.E. Smith). CoudRIED et al. (1985) trataram folhas de algodão com extrato aquoso de sementes de $A$. indica e observaram redução na oviposição de Bemisia tabaci (Genn.), e concentrações de 0,2 e $2 \%$ repeliram de modo semelhante a praga. Kirpal et al. (1986) também verificaram em diferentes extratos de $A$. indica alto efeito repelente e antialimentar, reduzindo significativamente a população de Brevicoryne brassicae em plantas de repolho.
Stein e Klingauf (1990) estudaram o efeito de extratos etanólicos de algumas plantas e verificaram que os extratos de Chrysanthemum cinerariaefolium e Persea americana proporcionaram, respectivamente, $100 \%$ e $74,8 \%$ de controle de $P$. xylostella.

De acordo com CHEN et al. (1996), extratos orgânicos de Melia azedarach causaram 93,5\% de redução na oviposição de $P$. xylostella na concentração de $4 \%$, sendo essa redução proporcional à concentração utilizada.

Torres (2000) analisou o efeito de extratos aquosos de plantas em relação a $P$. xylostella, constatando que a oviposição da praga foi diretamente correlacionada com o aumento das concentrações dos extratos, independentemente da espécie vegetal utilizada, e que o efeito repelente se acentua com a quantidade de substâncias bioativas extraídas e existente em cada extrato; os extratos de Aspidosperma pyrifolium, A. indica e Cissampelos aff. glaberrima foram os mais repelentes.

Devido à importância da traça-das-crucíferas, que causam perdas significativas na cultura da couve, objetivou-se com este trabalho avaliar o efeito de extratos aquosos de 18 espécies de plantas, aplicados sobre folhas de couve, na deterrência para a oviposição de P. xylostella, em condições de laboratório.

\section{MATERIAL E MÉTODOS}

A pesquisa foi desenvolvida em laboratório à temperatura de $25 \pm 1{ }^{\circ} \mathrm{C}$, umidade relativa de $74 \% \pm$ $5 \%$ e fotofase de 12 horas.

Sementes de couve, B. oleracea var. acephala, cultivar Georgia, foram semeadas em bandejas de isopor contendo substrato Plantmax ${ }^{\circledR}$, e mantidas em casa de vegetação. Após 30 dias, foram transplantadas para canteiro definitivo na Área Experimental do Departamento de Fitossanidade, recebendo tratos culturais padrão para a cultura (CAMARGo, 1992). Irrigações por aspersão foram realizadas quando necessário. 
Para o preparo dos extratos foram utilizados folhas, ramos, frutos e cascas de plantas (Tabela 1), coletadas no Campus da FCAV/UNESP - Jaboticabal (SP), com exceção de Trichilia pallida e de T. catigua, coletadas na Mata Santa Tereza, na cidade de Ribeirão Preto. Logo após a coleta, as partes dos vegetais foram colocadas para secagem em estufa à temperatura de 35 a $38^{\circ} \mathrm{C}$, por um período de 15 dias, até massa constante, e moídas em seguida com auxílio de moinho de facas, sendo o pó peneirado em peneira de $0,8 \mathrm{~mm}$.

No mesmo dia da moagem, foram preparadas suspensões contendo $10 \mathrm{~g}$ de cada espécie vegetal moída (Tabela 1) e $100 \mathrm{~mL}$ de água destilada, permanecendo em repouso por 12 horas, com o propósito de extrair os compostos hidrossolúveis. Decorrido esse tempo, coou-se usando tecido tipo 'voile', obtendo-se extratos na concentração (massa/ volume) de $10 \%$.

Após a obtenção dos extratos, discos de $8 \mathrm{~cm}$ de diâmetro de folhas de couve foram imersos em cada extrato por um período de um minuto.
A testemunha foi constituída por discos imersos em água destilada. Depois desse tempo, os discos foram colocados sobre papel toalha e deixados ao ar livre para perda do excesso de umidade superficial por cerca de uma hora, sendo em seguida divididos em quatro partes iguais, obtendo-se triângulos com dimensões e textura semelhantes.

Discos retirados das mesmas folhas de couve foram imersos em água destilada e usados como padrão no teste de deterrência. Formou-se, assim, um conjunto constituído por quatro triângulos dispostos alternadamente sobre papel filtro levemente umedecido com água destilada, sendo dois tratados com extratos e dois tratados com água destilada.

Foram feitas marcações no papel filtro, sob cada triângulo de folha de couve, para identificar as partes tratadas com os extratos e as partes tratadas com água, para posterior avaliação. Esse conjunto foi colocado sobre um disco de esponja, com o mesmo diâmetro do papel filtro, sustentado por um copo plástico, e transferido para gaiolas plásticas.

Tabela 1. Denominação e estrutura vegetal das plantas utilizadas na avaliação dos efeitos dos extratos aquosos na preferência para oviposição de Plutella xylostella. Jaboticabal (SP), 2004

\begin{tabular}{|c|c|c|c|}
\hline Nome científico & Nome comum & Família & Partes vegetais \\
\hline Achillea millefolium L. & Mil-folhas & Asteraceae & Folhas \\
\hline Azadirachta indica A. Juss. & Nim & Meliaceae & Folhas \\
\hline Bidens pilosa $\mathrm{L}$. & Picão-preto & Asteraceae & Ramos + Folhas + Frutos \\
\hline Bougainvillea glabra Choisy & Primavera & Nyctaginaceae & Folhas \\
\hline Chenopodium ambrosioides L. & Erva-de-santa-maria & Chenopodiaceae & Ramos + Folhas + Frutos \\
\hline $\begin{array}{l}\text { Datura suaveolens } \\
\text { Humb \& Bonpl. ex. Willd }\end{array}$ & Trombeteira & Solanaceae & Folhas \\
\hline $\begin{array}{l}\text { Enterolobium contortisilliquum } \\
\text { (Vell.) Morong }\end{array}$ & Tamboril & Mimosaceae & Frutos com sementes \\
\hline Mentha crispa L. & Hortelã & Lamiaceae & Folhas + Ramos \\
\hline Nicotiana tabacum L. & Fumo & Solanaceae & Folhas \\
\hline Piper nigrum L. & Pimenta-do-reino & Piperaceae & Folhas \\
\hline Plumbago capensis Thunb. & Plumbago & Plumbaginaceae & Folhas + Ramos \\
\hline Pothomorphe umbellata L. & Pariparoba & Piperaceae & Folhas \\
\hline Sapindus saponaria L. & Sabão-de-soldado & Sapindaceae & Folhas \\
\hline S. saponaria L. & Sabão-de-soldado & Sapindaceae & Frutos \\
\hline Solanum cernuum Vell. & Panacéia & Solanaceae & Folhas \\
\hline $\begin{array}{l}\text { Stryphnodendron adstringens } \\
\text { (Mart) Coville }\end{array}$ & Barbatimão & Mimosaceae & Casca \\
\hline Symphytum officinale L. & Confrei & Boraginaceae & Folhas \\
\hline Trichilia catigua A. Juss. & Trichilia & Meliaceae & Folhas \\
\hline T. catigua A. Juss. & Trichilia & Meliaceae & Ramos \\
\hline T. pallida Sw. & Trichilia & Meliaceae & Folhas \\
\hline T. pallida Sw. & Trichilia & Meliaceae & Ramos \\
\hline
\end{tabular}


Em cada gaiola, colocou-se um casal de $P$. xylostella com até 12 horas de idade, proveniente de criação feita em laboratório, e mantido por quatro dias para oviposição, sendo realizada, diariamente, a contagem dos ovos em cada um dos triângulos e sua substituição por outro. O efeito deterrente dos extratos foi avaliado através da fórmula: $\mathrm{PD}=(\mathrm{NC}-\mathrm{NT}) /(\mathrm{NC}$ + NT) x 100, adaptada de Obeng-Ofori (1995), sendo $\mathrm{PD}$, a porcentagem média de deterrência; NC, o número de ovos no tratamento com água destilada; e NT, o número de ovos em cada tratamento com extrato. Foi atribuída a seguinte classificação: Deterrente PD $>0$ e Neutro: $P D<0$. Cada repetição foi constituída por uma gaiola contendo um casal da praga.

O delineamento experimental utilizado foi inteiramente casualizado, com 22 tratamentos e quatro repetições. Com relação à testemunha nas quatro repetições, foram colocados os triângulos de folhas alternados, apenas imersos em água destilada, aplicando-se a fórmula de PD, considerando-se como $\mathrm{NC}$, os dois triângulos onde se encontra o maior número de insetos. Os resultados foram submetidos à análise de variância e as médias comparadas pelo teste de Tukey $(\mathrm{P}<0,05)$, utilizando-se o programa SANEST (versão 3.0).

\section{RESULTADOS E DISCUSSÃO}

Os extratos promoveram efeito deterrente na oviposição de P. xylostella, exceto o extrato de Stryphnodendron adstringens, que não diferiu da testemunha tratada apenas com água, porém apresentou deterrência de 46,9\% (Tabela 2). Houve uniformidade da postura sobre os discos de folha de couve imersos em água destilada, uma vez que a porcentagem de deterrência (PD) foi igual a 6,9\%, significando que a quantidade de ovos colocados foi similar nos triângulos de folhas de couve expostos à oviposição da praga, resultando em índice baixo em relação aos demais tratamentos.

Tabela 2. Porcentagem média $( \pm \mathrm{EP})$ de deterrência para oviposição de Plutella xylostella em discos de couve tratados com extratos aquosos de espécies vegetais à concentração de $10 \%$ (massa/volume). T $\left({ }^{\circ} \mathrm{C}\right)=25 \pm 1$; UR $(\%)=74 \pm 5$; fotofase $=12$ horas. Jaboticabal (SP), 2004

\begin{tabular}{|c|c|}
\hline Tratamento & Porcentagem de deterrência (PD) \\
\hline Enterolobium contortisilliquum & $100,00 \mathrm{a}$ \\
\hline Sapindus saponaria (frutos) & $100,00 \mathrm{a}$ \\
\hline Trichilia pallida (folhas) & $100,00 \mathrm{a}$ \\
\hline Nicotiana tabacum & $99,5 \pm 0,34 a b$ \\
\hline Chenopodium ambrosioides & $98,6 \pm 0,61 \mathrm{ab}$ \\
\hline Trichilia pallida (ramos) & $95,6 \pm 1,93 \mathrm{ab}$ \\
\hline Bougainvillea glabra & $95,3 \pm 0,86 a b$ \\
\hline Achillea millefolium & $89,7 \pm 1,47 \mathrm{abc}$ \\
\hline Azadirachta indica & $89,1 \pm 2,30 \mathrm{abc}$ \\
\hline Datura suaveolens & $88,9 \pm 1,76 \mathrm{abc}$ \\
\hline Symphytum officinale & $86,6 \pm 3,81 \mathrm{abc}$ \\
\hline Solanum cernuum & $86,0 \pm 2,72 a b c$ \\
\hline Trichilia catigua (ramos) & $79,6 \pm 2,93 \mathrm{abc}$ \\
\hline Pothomorphe umbellata & $78,9 \pm 5,29 \mathrm{abc}$ \\
\hline Trichilia catigua (folhas) & $74,7 \pm 7,14 \mathrm{abc}$ \\
\hline Bidens pilosa & $73,1 \pm 9,23 \mathrm{abc}$ \\
\hline Plumbago capensis & $72,5 \pm 7,40 \mathrm{abc}$ \\
\hline Piper nigrum & $64,1 \pm 9,23 \mathrm{abc}$ \\
\hline Mentha crispa & $58,9 \pm 11,67 \mathrm{abc}$ \\
\hline Sapindus saponaria (folhas) & $54,5 \pm 12,52 \mathrm{bc}$ \\
\hline Stryphnodendron adstringens & $46,9 \pm 7,23 \mathrm{~cd}$ \\
\hline Testemunha & $6,9 \pm 12,82 \mathrm{~d}$ \\
\hline
\end{tabular}

Médias seguidas pela mesma letra, não diferem entre si pelo teste de Tukey, a 5\% de probabilidade. 
Os extratos de E. contortisilliquum, S. saponaria (frutos) e T. pallida (folhas) foram os que obtiveram os melhores resultados, com 100\% de deterrência (Tabela 2), seguido dos extratos de $N$. tabacum, C. ambrosioides, T. pallida (ramos) e Bougainvillea glabra, com deterrência acima de $95 \%$. Os demais extratos também possuem bom efeito deterrente, entre $89,7 \%$ ( $A$. millefolium) e $54,5 \%$ (S. saponaria - folhas).

Essa deterrência também foi constatada por outros autores, como Coudriet et al. (1985), que trataram folhas de algodão com extrato aquoso de sementes de $A$. indica e observaram redução na oviposição de B. tabaci. Segundo GUPTA e THORSTEINSON (1960), a oviposição de diversos lepidópteros geralmente é mediada por mecanismos sensoriais, mecano e químio-receptores. Devaraj e SRILATHa (1993) estudaram as propriedades repelentes de extratos contra Corcyra cephalonica e constataram que o extrato de eucalipto foi o mais repelente, seguido por Cymbopogon, mostarda, nim e datura.

A ação deterrente de extratos vegetais na oviposição de insetos ainda é pouco conhecida, sendo poucos os trabalhos que mencionam esse fato. TORRES (2000) avaliou o efeito de extratos aquosos de cinco espécies vegetais na oviposição de $P$. xylostella, em diferentes concentrações, em que o extrato da casca de A. pyrifolium continha porcentagem de repelência de $56,4 \%$ à concentração de $7,5 \%$, sendo superior aos extratos das demais plantas.

À medida que se aumentou a concentração, independentemente da espécie vegetal utilizada, aumentou a percentagem de repelência, visto que o efeito repelente se acentua com a quantidade de substâncias bioativas extraídas e existentes em cada extrato.

No presente estudo, apesar de algumas plantas não terem influenciado a oviposição de P. xylostella, não devem ser descartadas. Deve-se testar outros meios de extração dos princípios ativos existentes nas plantas, pois Roel et al. (2000) verificaram diferentes resultados entre os diversos solventes utilizados. Também Stein e Klingauf (1990), estudando o efeito de extratos etanólicos e aquosos de folhas de Prosopis juliflora contra Myzys persicae e larvas de P. xylostella, observaram eficácia de $90 \%$ e $28 \%$ com extrato etanólico e $6 \%$ e $10 \%$ com extrato aquoso respectivamente. Também podem ser estudadas outras estruturas dessas plantas, pois podem ter diferentes concentrações dos princípios ativos, como $S$. saponaria neste trabalho, em que para os frutos, houve $100 \%$ de deterrência, e nas folhas, a redução foi de $54,5 \%$ na oviposição da praga.

\section{CONCLUSÕES}

1. Os extratos avaliados proporcionaram efeito deterrente na oviposição de $P$. xylostella, exceto o de $S$. adstringens.

2. Os extratos de E. contortisilliquum, S. saponaria (frutos) e T. pallida (folhas) causaram 100\% de deterrência na oviposição de P. xylostella.

\section{AGRADECIMENTOS}

Ao CNPq (Conselho Nacional de Desenvolvimento Científico e Tecnológico), pela bolsa de Produtividade em Pesquisa, concedida ao segundo autor.

\section{REFERÊNCIAS}

CAMARGO, L. S. As hortaliças e seu cultivo. 3. ed. São Paulo: Fundação Cargill, 1992. 252p.

CHEN, C.; CHANG, S.; CHENG, L.;HOU, R. F. Deterrent effect of the chinaberry extract on oviposition of the diamondback moth, Plutella xylostella (L.) (Lep. Yponomeutidae). Journal Applied Entomology, Berlin, v.120, p.165-169, 1996.

COUDRIET, D. L.; PRABHAKER, N.; MEYERDIRK, D. E. Sweetpotato whitefly (Homoptera: Aleyrodidae): effects of neem-seed extract on oviposition and immature stages. Environmental Entomology, Lanhan, v.14, p.776-779, 1985.

DEVARAJ, K. C.; SRILATHA, G. M. Antifeedant and repellent properties of certain plant extracts against the rice moth, Corcyra cephalonica St. Botanical Pesticides in Integrated Pest Management, Bangalore, v.8, p.159-165, 1993.

FRANÇA, F.H.; CORDEIRO, C.M.T.; GIORDANO, L.B.; RESENDE A.M. Controle da traça das crucíferas em repolho. Horticultura Brasileira, Brasília, v.3, n.2, p.50-51, 1985.

FRANCO, G. Tabela de composição química de alimentos. 3.ed. Rio de Janeiro: Serviço de Alimentação da Previdência Social, 1960. 194p.

GALLO, D.; NAKANO, O.; SILVEIRA NETO, S.; CARVALHO, R.P.L.; BATISTA, G.C.; BERTI FILHO, E.; PARRA, J.R.P.; ZUCCHI, R.A.; ALVES, S.B.; VENDRAMIM, J.D.; MARCHINI, L.C.; LOPES, J.R.S.; OMOTO, C. Entomologia agrícola. Piracicaba: FEALQ, 2002. 920 p.

GUPTA, P. D.; THORSTEINSON, A. F. Food plant relationships of the diamondback moth (Plutella maculipennis Curt.). II. Sensory regulation of oviposition of the adult female. Entomologia Experimentalis et Applicata, Dordrecht, v.3, p.305-314, 1960. 
KIRPAL, S.; SHAMA, P. L.; SINGH, K. Studies on the antifeedant and repellent qualities on neem (Azadirachta indica) against aphid (Brevicoryne brassicae L.) on cauliflower and cabbage. Research and Development Reporter, Solan, v.3, n.1, p.33-35, 1986.

MARANHÃO, E. A. de A.; LIMA, M. P. L. de; MARANHÃO, E. H. de A.; LYRA FILHO, H. P. Flutuação populacional da traça das crucíferas, em couve, na zona da Mata de Pernambuco. Horticultura Brasileira, Brasília, v.16, n.1, 1998.

OBENG-OFORI, D. Plant oils as grain protectants against infestations of Cryptolestes pusillus and Rhyzopertha dominica in stored grain. Entomologia Experimentalis et Applicata, Doidrecht, v.77, p.133-139, 1995.

OOI, P.A.C.; KELDERMAN, W. The biology of three common pests of cabagges in Cameron Highlands, Malaysia. Malaysian Agricultural Journal, Kuala Lumpur, v.52, n.1, p.85-101, 1979.

ROEL, A. R.; VENDRAMIM, J. D.; FRIGHETTO, R. T. S.; FRIGHETTO, N. Atividade tóxica de extratos orgânicos de Trichilia pallida Swartz (Meliaceae) sobre Spodoptera frugiperda (J. E. Smith). Anais da Sociedade Entomológica do Brasil, Londrina, v.29, n.4, p.799-808, 2000.
SCHMUTTERER, H. Properties and potential of natural pesticides from the neem tree, Azadirachta indica. Annual Review Entomology, Palo Alto, v.35, p.271-297, 1990.

STEIN, U.; KLINGAUF, F. Insecticidal effect of plant extracts from tropical and subtropical species. Traditional methods are good as long as they are effective. Journal of Applied Entomology, Berlin, v.110, n. 2, p.160-166, 1990.

TORRES, A. L. Efeito de extratos aquosos de plantas na biologia de Plutella xylostella (L. 1758) (Lepidoptera: Plutellidae). 58f. 2000. Dissertação (Mestrado em Agronomia) Universidade Federal Rural de Pernambuco, Recife.

VILLASBOAS, G. L.; CASTELOBRANCO, M.; GUIMARÃES, A. L. Controle químico da traça das crucíferas em repolho do Distrito Federal. Horticultura Brasileira, Brasília, v.8, n.2, p.10-11, 1990. 